\title{
The MJA, MDA National, Nossal Global Health Prize: broadening our horizons
}

lobal health is, by definition, an area of great breadth and depth. There is enormous diversity in health systems and health outcomes, with life expectancy at birth varying from 82-84 years in Australia and Japan to less than 55 years in more than 20 African countries. Despite this diversity and difference, communicable diseases such as HIV and severe acute respiratory syndrome, and our more recent understanding of the global industrial vectors of noncommunicable diseases such as the tobacco industry, show that our health is becoming increasingly interconnected and interdependent.

How should Australia and our doctors be responding? Are we investing sufficiently in undergraduate and postgraduate medical education to have enough doctors who are highly competent in global health? Can we get better insight into our own medical and public health practices in Australia by improving our understanding of the world around us?

In recent times, the interest and contribution to global health issues by young graduates and medical students has grown, seemingly exponentially. Examples include:

- the establishment of the go-to website Global Health Gateway (http://www.globalhealthgateway.org.au/), an online access point to global health vocational and training information and opportunities for people in Australia and New Zealand;

- the number of students and young doctors working in resource-constrained settings;

- the development and publication (by this Journal) of $A$ guide to working abroad for Australian medical students and junior doctors (http://ama.com.au/gwa);

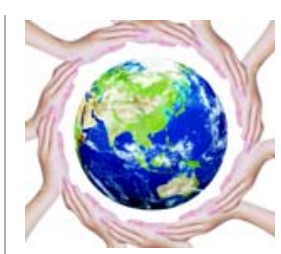

A Rob Moodie MB BS, MPH, Professor of Global Health

Nossal Institute for Global Health, University of Melbourne, Melbourne, VIC.

r.moodie@ unimelb.edu.au

doi: 10.5694/mjal2.11340
- the first Global Ideas Forum, which was held in Melbourne in August 2012; and

- the series of very successful global health conferences run annually by the Australian Medical Students' Association.

This interest is also manifest in the increasing demand by young medical graduates that overseas training in developing countries be recognised and encouraged as part of their specialist training.

To better understand and encourage this burgeoning interest in global health, the Nossal Institute for Global Health, MDA National and the Medical Journal of Australia established the Global Health essay competition. Our aim is not only to stimulate interest in this important area of health and medicine, but to also highlight the challenges and reflective learning emanating from the experiences of both medical students and graduates.

In this, the first year of the competition, we received 20 entries from graduates and 14 from students. We chose a winner and two runners-up from each category, all of which will be published in the MJA. We were delighted with the ideas and learnings evident in the essays, and moved by the intensely personal, reflective and insightful nature of the best of them.

I would like to thank MDA National and the $M J A$, and, in particular, the former editor Annette Katelaris, without whom this annual competition might not have seen the light of day. Thanks also to my fellow panel member Charles Guest.

We hope that you will read these contributions and be as challenged, moved and inspired as we have been. 